\title{
Optimal sigmoidal tuning curves for intensity encoding sensory neurons with quasi-Poisson variability Mark D McDonnell ${ }^{* 1}$ and Nigel G Stocks²
}

\author{
Address: ${ }^{1}$ Institute for Telecommunications Research, University of South Australia, Mawson Lakes, SA 5095, Australia and ${ }^{2}$ School of Engineering, \\ University of Warwick, Coventry CV4 7AL, UK \\ Email: Mark D McDonnell* - mark.mcdonnell@unisa.edu.au \\ * Corresponding author
}

from Seventeenth Annual Computational Neuroscience Meeting: CNS*2008

Portland, OR, USA. 19-24 July 2008

Published: II July 2008

BMC Neuroscience 2008, 9(SuppI I):PII7 doi:I0.I I86/I47I-2202-9-SI-PI I7

This abstract is available from: http://www.biomedcentral.com/I47I-2202/9/SI/PII7

(c) 2008 McDonnell and Stocks; licensee BioMed Central Ltd.

\section{Background}

Rate-coding neurons are often characterized by their tuning curve, that is, the average firing rate, $T(x)$, as a function of stimulus intensity, $x$. However the substantial natural variability in firing rate that often occurs for a fixed stimulus provides a limitation on the fidelity of firing rate encoding of stimuli. Consequently, stimulus-dependent variance in firing rate, $\mathrm{V}(\mathrm{x})$, is crucial in studies of tuning curve optimality. Information theory can be used to quantify such limits and to address the question of finding the tuning curve that maximizes information rate [1].

Firing activity is often modeled as a Poisson point process, such that $V(x)=T(x)$. However, this assumption can break down for intensity encoding neurons with monotonically non-decreasing (e.g. sigmoidal) tuning curves, such as primary afferent auditory nerve fibers, where refractoriness can cause firing rate saturation. As the rate nears this point, variability decreases, and to a first approximation becomes binomial rather than Poisson, so that $V(x)$ varies quadratically with $T(x)$. Such neurons are sometimes called quasi-Poisson.

\section{Results}

We have derived a sufficient condition for achieving maximum Shannon mutual information between stimulus intensity and firing rate when the variability is quasi-Poisson such that $V(x)=s^{2} T(x)(1-T(x))$, and $s$ is small [2]. The sufficient condition leads to analytical expressions for two ways to achieve maximize mutual information: (i) an optimal monotonically non-decreasing tuning curve for any given stimulus distribution and (ii) an optimal stimulus for any given monotonically non-decreasing tuning curve [2].

The optimal tuning curve for a stimulus with cumulative distribution function $F_{x}(x)$ is $T^{o}(x)=0.5-0.5 \cos \left(\pi F_{x}(x)\right)$, while for a tuning curve $T(x)$, the optimal probability density function of the stimulus is $f_{x}^{o}(x)=(d T(x) / d x) /(\pi$ $\left.(T(x)(1-T(x)))^{0.5}\right)$. Our derivation also provides an expression for the reduction in mutual information when the tuning curve and stimulus distribution are not optimally matched [2]. This expression is a function of the relative entropy between the stimulus distribution, and a distribution known as Jeffrey's prior. The derivation makes use of a relationship between Shannon mutual information and Fisher information discussed, for example, in [1].

\section{Discussion}

Unlike neurons with a 'preferred stimulus' (unimodal tuning curves), optimality conditions for neurons where firing rates increase monotonically with stimulus intensity (e.g. sigmoidally) have received little attention. A notable exception is $[3,4]$, which maximizes Fisher information, and considers only Poisson variability. In contrast, we maximize mutual information, and consider quasi-Poisson variability. This leads to a very versatile analytical solution that allows for refractoriness. A limitation to be addressed in future work is how well the quadratic relationship $V(x)=s^{2} T(x)(1-T(x))$ compares with measured variability. Finally, while we assume small s, our solution provides a lower bound to the achievable 
mutual information for larger s, and is hence a worst-case scenario.

\section{Acknowledgements}

This work was funded by Australian Research Council grant DP0770747, and EPSRC grant EP/C523334/I, and we gratefully acknowledge this support. We also thank Emilio Salinas and Simon Durrant for valuable discussions.

\section{References}

I. Brunel N, Nadal J: Mutual information, Fisher information and population coding. Neural Computation 1998, 10:173 I-1757.

2. McDonnell MD, Stocks NG: Maximally informative stimuli and tuning curves for sigmoidal rate-coding neurons with quasipoisson variability. Submitted to Physical Review Letters. arXiv:0802.1570vl.

3. Bethge M, Rotermund D, Pawelzik K: Optimal short-term population coding: When Fisher information fails. Neural Computation 2002, 14:2317-235I.

4. Bethge $M$, Rotermund D, Pawelzik K: Optimal neural rate coding leads to bimodal firing rate distributions. Network: Computation in Neural Systems 2003, 14:303-319.

Publish with Bio Med Central and every scientist can read your work free of charge

"BioMed Central will be the most significant development for disseminating the results of biomedical research in our lifetime. "

Sir Paul Nurse, Cancer Research UK

Your research papers will be:

- available free of charge to the entire biomedical community

- peer reviewed and published immediately upon acceptance

- cited in PubMed and archived on PubMed Central

- yours - you keep the copyright

Submit your manuscript here:

http://www.biomedcentral.com/info/publishing_adv.asp 